\title{
Simple numerical calculation method of flow velocity on roof of train running in tunnel
}

\author{
Katsuhiro KIKUCHI*, Yuhei NOGUCHI*, Koji NAKADE** and Shinya MASHIMO*** \\ *Vehicle Aerodynamics, Railway Technical Research Institute \\ ${ }^{* *}$ Computational Mechanics, Railway Technical Research Institute \\ 2-8-38 Hikari-cho, Kokubunji-shi, Tokyo 185-8540, Japan \\ E-mail: kikuchi.katsuhiro.74@rtri.or.jp \\ ***Rolling Stock Design Office, West Japan Railway Company \\ 2-4-24 Shibata, Kita-ku, Osaka 530-8341, Japan
}

Received: 20 October 2017; Revised: 4 February 2018; Accepted: 23 May 2018

\begin{abstract}
A pantograph mounted on a high-speed train roof is exposed to high-speed air flow while traveling, and lift force is generated in the pantograph. To ensure high current collecting performance of the pantograph, it is important to appropriately adjust the lift force generated in the pantograph. The flow velocity along the roof of a train has a significant influence on the lift force. In this study, we proposed a simple calculation method that enabled us to easily calculate the flow on the train roof in a tunnel using a personal computer. To validate the effectiveness of the proposed method, model experiments were carried out and calculation and experimental results were compared. Furthermore, the influence of hydraulic friction coefficients on train roof flow velocity was examined using the proposed method, which was not time consuming.
\end{abstract}

Keywords : Train, Tunnel, Pantograph, Train roof flow, Simple calculation method

\section{Introduction}

A pantograph is installed on a roof of a train to receive electric power from overhead contact line facilities. In the case of high-speed trains, the pantograph is exposed to high-speed air flow and lift force is generated in the pantograph. The lift force generated in the pantograph must be stable so that the pantograph can exhibit high current collecting performance in high-speed scenarios (Manabe, 1988; Suzuki et al., 2008; Ikeda, 2013; Flueckiger et al., 2013; Mitsumoji at al., 2016). The pantograph head is designed to have a blunt shape, which causes flow separation at a specific position of the pantograph head so that the lift force do not change significantly.

Pressure fluctuation and flow velocity fluctuation occur when a train runs in a tunnel at high speed. Between these two phenomena, extensive research has been carried out on pressure fluctuation to maintain the comfort of passengers in a passenger cabin, to design a ventilation system inside a vehicle, and to evaluate the safety of equipment in tunnels. In contrast, extremely few studies have been conducted on flow velocity until now. This is because of the following reasons: 1) The analysis of flow velocity fluctuation was less urgent than that of pressure fluctuation. 2) Pressure is almost uniform within a tunnel cross section; however, flow velocity has a distribution within the tunnel cross section. Thus, on-site measurement becomes large scale. 3) As there is relative motion between a tunnel and a train, it is difficult to measure the flow velocity around a train model through a model experiment.

The flow velocity toward the pantograph when the train travels in a tunnel is larger than that when the train runs in an open section. The lift force acting at the pantograph head is proportional to the square of the flow velocity toward the pantograph head. Therefore, the flow velocity toward the pantograph head is an important parameter that affects the lift force. However, it is difficult to accurately predict the flow velocity at the pantograph head under various train and tunnel conditions when the train travels in a tunnel, based on the data obtained from an on-track test. In contrast, based on the one-dimensional pressure fluctuation simulation for a tunnel (Yamamoto, 1968; Iida and Maeda, 1990; Kajiyama et al., 1994), the flow velocity toward the pantograph head can be estimated under various train and tunnel conditions. However, 
the flow velocity calculated by the one-dimensional pressure fluctuation simulation is smaller than that obtained through on-track measurement. This difference can be attributed to the following factor: The flow velocity distribution in a boundary layer in the tunnel cross section is not considered in the one-dimensional pressure fluctuation simulation. To examine the effect of the boundary layer, several researchers are analyzing the flow around a train running in a tunnel using three-dimensional numerical fluid simulation. However, a large-scale computer and dedicated software are necessary to carry out three-dimensional numerical fluid simulation. Furthermore, performing such numerical analysis requires enormous cost and time. Although the calculation cost of the one-dimensional pressure fluctuation simulation is smaller compared to three-dimensional numerical fluid simulation, a large-scale computer is still necessary to perform computation. Thus, three-dimensional numerical fluid analysis and the one-dimensional pressure fluctuation simulation cannot be easily used for the desktop calculation of the flow on a train roof.

Therefore, in this study, we proposed a simple calculation method that enabled us to easily calculate the flow on the train roof in a tunnel using a personal computer, including the effect of the boundary layer. To demonstrate the effectiveness of the proposed method, we performed model experiments and compared calculation results and experimental results. Furthermore, we investigated the influence of hydraulic friction coefficients on train roof flow velocity using the proposed method, which was not time consuming.

\section{Outline of flow velocity distribution on a roof of a train running in a tunnel and problems of one- dimensional pressure fluctuation simulation}

We present the on-track measurement results obtained by other researchers and an outline of the flow field on the roof of a train running in a tunnel. Then, we describe the problems of the one-dimensional pressure fluctuation simulation, which is widely used for predicting flow velocity when a train runs in a tunnel.

To understand the flow field on the roof of a train running in a tunnel, a measurement method has been developed using a three-hole tube embedded in the pantograph head and using the pantograph head as the flow direction/flow velocity sensor (Ikeda et al., 2011). Based on the on-track measurement results obtained using the above-mentioned method, the following issues were clarified: The flow velocity toward the pantograph head is approximately equal to the running speed of the train in an open section. However, on average, the flow velocity toward the pantograph head increases by 1.2-1.3 times the running speed of the train in a tunnel section. Under certain conditions, the flow velocity toward the pantograph head increases by up to approximately 1.6 times the running speed of the train. A comparison between the one-dimensional pressure fluctuation simulation result and the on-track measurement result of the flow velocity toward the pantograph head is shown in Fig. 1. It can be seen that the trend of the one-dimensional pressure fluctuation simulation result is almost in agreement with that of the on-track measurement result in terms of the qualitative tendency, such as how the flow velocity toward the pantograph head changes. However, the one-dimensional pressure fluctuation simulation assumes that the flow velocity distribution in the cross section of the tunnel is uniform (spatial average flow velocity) and treats the flow in the tunnel as one-dimensional flow. Therefore, the one-dimensional pressure fluctuation simulation does not consider the influence of the boundary layer on the train roof and on the tunnel wall (see Fig. 2) when calculating the flow velocity toward the pantograph head. Accordingly, an increase in the flow velocity owing to the displacement effect of the boundary layer is underestimated. The phenomenon mentioned above may be reversed depending on the reference point of the flow velocity and the situation of development of the boundary layer. That is, the flow velocity obtained by the one-dimensional pressure variation simulation may become faster than the actual flow velocity when the measuring point is near the rear of the long train, where the boundary layer sufficiently develops. When the reference point of the flow velocity is within the boundary layer, the flow velocity at the reference point becomes lower than the spatial average flow velocity. 


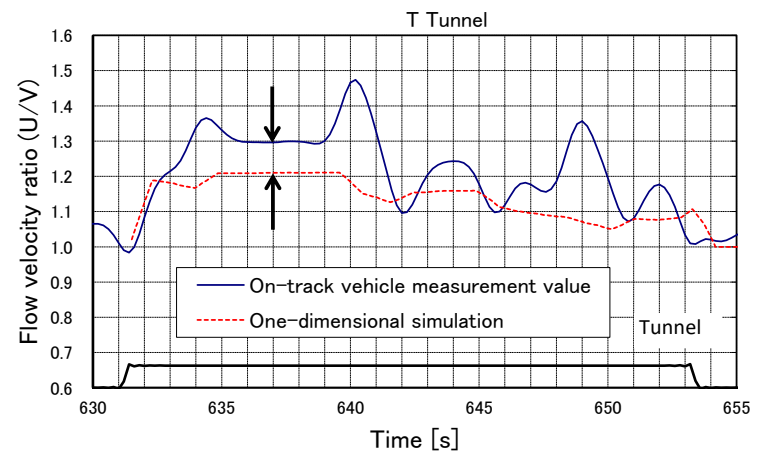

Fig. 1 Comparison between the flow obtained by onedimensional simulation and that obtained by on-track measurement when the train travels in a tunnel

Running speed: $V=300 \mathrm{~km} / \mathrm{h}$, A-series train, T tunnel, Tunnel length: $L=1822 \mathrm{~m}$

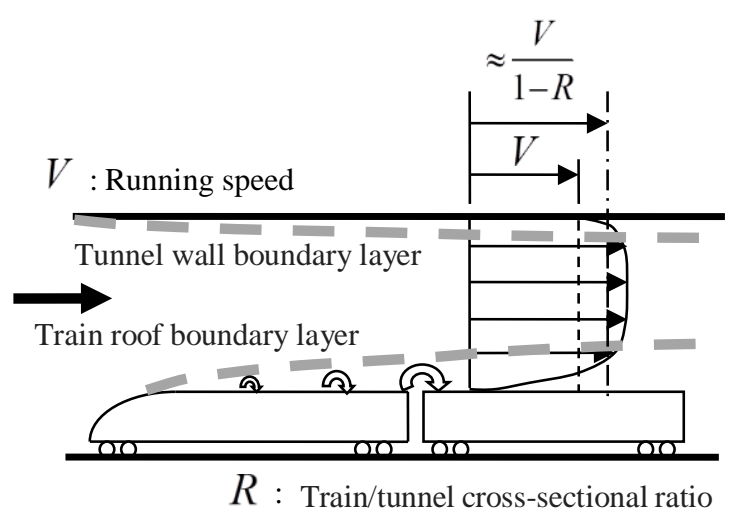

Fig. 2 Schematic diagram of flow on the train roof in a tunnel with the coordinate system fixed to the train

\section{Simple calculation method}

Three-dimensional numerical fluid simulation can consider the detailed shapes of trains and tunnels and incorporate the effects of boundary layers into calculation. However, it requires dedicated software and a large-scale computer. Although the one-dimensional pressure fluctuation simulation requires less computational cost than three-dimensional numerical fluid simulation, it still requires a large-scale computer and cannot be easily used on site. In this paper, we propose a simple desktop calculation method that does not require dedicated software or a high-performance computer. In this simple calculation method, the targeted calculation accuracy is set to $70-80 \%$ of that of the experimental results. This calculation accuracy is comparable to that of the one-dimensional pressure fluctuation simulation. The onedimensional pressure fluctuation simulation is used as a standard tool for predicting the pressure and flow velocity generated when a train travels through a tunnel (Saito et al., 2011; Yamauchi et al., 2016). Furthermore, the onedimensional pressure fluctuation simulation is used not only for studying the phenomena in the tunnel but also for studying the thermal environment and ventilation in the subway stations (Kajiyama, 2003), and the aerodynamic phenomena in Shinkansen stations (Minegishi et al., 2008). The flow velocity predicted using the one-dimensional pressure fluctuation simulation is sufficiently practical in terms of engineering. The simple calculation method proposed in this paper provides even more engineering practicality compared to the one-dimensional pressure fluctuation simulation for predicting flow velocity in the following aspects: calculation accuracy is almost the same, a large-scale computer is not required, calculation time is short, and the flow velocity distribution can be taken into account.

We adopt Earnshaw's method (Earnshaw, 1970), which approximates the flow on a train roof with two boundary layers. In Earnshaw's method, it is assumed that hydraulic friction coefficients depend only on $\delta / k$, where $\delta$ is the thickness of the boundary layer and $k$ is the average roughness height, which is considered to be known. In contrast, in the simple calculation method, it is assumed that hydraulic friction coefficients also depend on $u_{\tau} k / v$, where $u_{\tau}$ is friction velocity and $v$ is kinetic viscosity. The hydraulic friction coefficients are obtained using Nikuradse's frictional resistance formula (Nikuradse, 1950) in the simple calculation method. The train side pressure gradient is required for calculating the flow velocity on the train roof. This gradient is obtained from its relationship with the flow velocity in front of the train. In Earnshaw's method, the train side pressure gradient is obtained by assuming that the flow velocity in front of the train is known. In contrast, in the simple calculation method, the train side pressure gradient is obtained by calculating the flow velocity in front of the train using Yamamoto's one-dimensional incompressible steady-state model (Yamamoto, 1973). 


\section{1 Calculation method}

\section{1. 1 Calculation model}

The coordinate system is fixed to the train. Let $x$ be the main flow direction (opposite to the traveling direction of the train) and let $y$ be the direction from the upper surface of the train roof to the surface of the tunnel wall. As models for representing a train traveling in a tunnel, the tunnel and train are treated as circular pipes (Fig. 3). The central axes of the two circular pipes are coincident. The flow between the two circular pipes (annular flow) is to be calculated. Let $G$ be the distance between the two circular pipes, $\delta^{\prime}$ be the thickness of the boundary layer on the train roof, and $\delta$ be the thickness of the boundary layer on the tunnel wall (Fig. 3). The procedure of the simple calculation method is outlined below. Consider two circular pipes whose diameters are $2 \delta$ and $2 \delta^{\prime}$, respectively (Fig. 4). The flow velocity distributions in the boundary layers for the respective circular pipes are calculated. $\delta^{\prime}$ is changed while satisfying $\delta+\delta^{\prime}=G$, so that the velocities at the center positions in the respective circular pipes coincide, and these calculations are repeated.

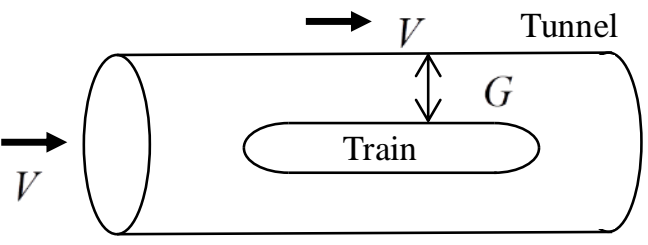

(a) Schematic of calculation model

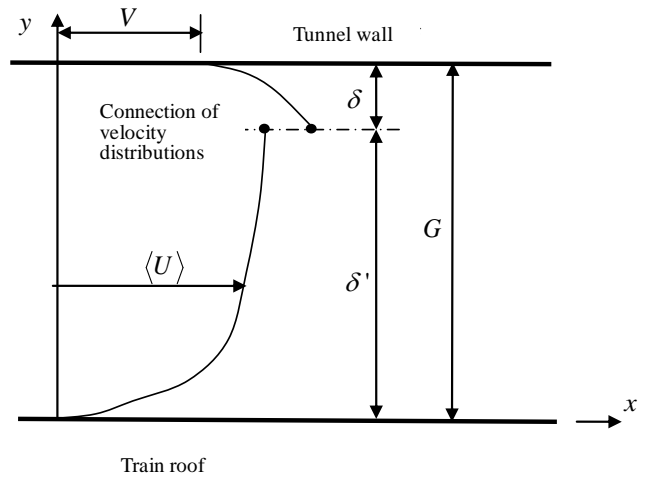

(b) Flow velocity distribution between circular pipes

Fig.3 Schematic of calculation model and flow velocity distribution
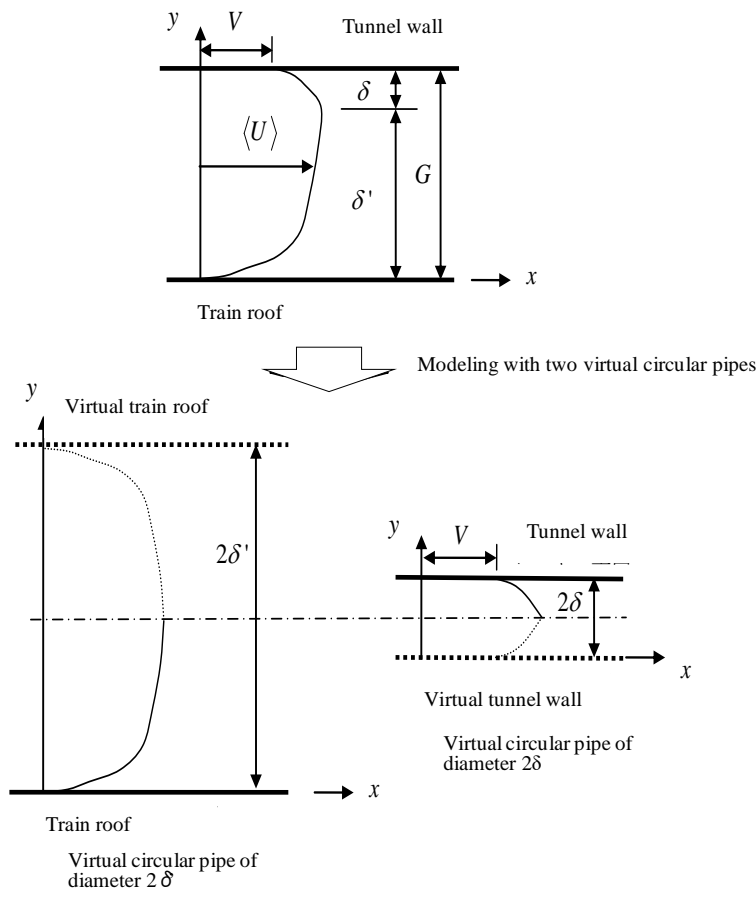

Fig. 4 Modeling with two virtual circular pipes

It is assumed that the circular pipe that represents the train is stationary while the circular pipe that represents the tunnel moves in the direction opposite to the traveling direction of the train at the running speed of the train. Furthermore, it is assumed that the flow between the two pipes forms a fully developed turbulent flow field. However, according to the results presented by Noguchi et al., 2015, the flow on the train roof at the position $160 \mathrm{~m}$ (sixth vehicle) away from the nose of the train does not form the fully developed turbulent flow field. Therefore, the model that approximates the flow between the two circular pipes with two fully developed boundary layers will include errors. However, we believe that it is possible to assume that the flow over the train roof forms the fully developed turbulent flow field if the accuracy of the calculation is set to $70-80 \%$ of that of the experimental results.

It is assumed that the flow between the two circular pipes satisfies the following conditions.

(a) The flow is statistically steady and incompressible.

(b) The flow between the two circular pipes is essentially flow between parallel plates, and the pressure gradient, $d p_{w} / d x$, is constant.

(c) The distribution of the average flow velocity between the two circular pipes can be represented by the log law.

In condition (b), flow between parallel plates (two-dimensional flow) is assumed. Because we have assumed the 
tunnel and train to be two circular pipes, we should assume circular pipe flow rather than two-dimensional flow. However, for simplicity, we calculate the boundary layer by assuming the target flow as two-dimensional flow, as stated in condition (b).

From condition (b), the equation of motion in the flow direction is (Pope, 2000)

$$
\frac{d \tau}{d y}=\frac{d p_{w}}{d x}
$$

where $\tau$ is the shear stress at position $y$ away from the train roof, $p_{w}$ is the average pressure on the wall surface, and $d p_{w} / d x$ is the pressure gradient.

From condition (c), the vertical distribution ( $y$ direction) of the ensemble-averaged flow velocity $\langle U\rangle$ in consideration of the average roughness height, $k$, is expressed as (Pope, 2000)

$$
\langle U\rangle=u_{\tau}\left(\frac{1}{\kappa} \ln \frac{y}{k}+C\right),
$$

where $\kappa$ is Karman's constant, $C$ is an additional constant that depends on $u_{\tau} k / v$, and $u_{\tau}$ is the friction velocity defined by

$$
u_{\tau}=\sqrt{\frac{\left|\tau_{w}\right|}{\rho}}
$$

where $\tau_{w}$ is the wall shear stress and $\rho$ is the air density.

We apply Eq. (2), which represents the ensemble-averaged flow velocity (hereafter, we simply refer to it as flow velocity), to the flow between the two circular pipes. Then, we obtain

$$
\left\{\begin{aligned}
\langle U\rangle & =u_{\tau}{ }^{\prime}\left(\frac{1}{\kappa} \ln \frac{y}{k^{\prime}}+C^{\prime}\right) \quad k^{\prime} \leq y<\delta^{\prime} \\
& =V+u_{\tau}\left(\frac{1}{\kappa} \ln \frac{(G-y)}{k}+C\right) \quad G-\delta \leq y \leq G-k
\end{aligned}\right.
$$

where the variables with and without the prime are the quantities related to the boundary layers on the train roof and tunnel wall, respectively. When the distributions of the respective flow velocities at the boundary layer edges of the train roof and tunnel wall coincide with each other, the following expression is satisfied:

$$
u_{\tau}{ }^{\prime}\left(\frac{1}{\kappa} \ln \frac{\delta^{\prime}}{k^{\prime}}+C^{\prime}\right)=V+u_{\tau}\left(\frac{1}{\kappa} \ln \frac{\delta}{k}+C\right) .
$$

From Eq. (1), the pressure gradient and the two shear stresses on the wall surfaces (the surfaces of the train roof and tunnel wall) are related by the following equation:

$$
\frac{d p_{w}}{d x}=\frac{\tau_{w}-\tau_{w}{ }^{\prime}}{G} .
$$

Further assumptions are required to determine the flow velocity distribution of the two boundary layers. For the two flow velocity distributions to be smoothly connected, the gradient of the flow distribution must be zero at the boundary layer edge. However, the log law does not hold at the boundary layer edge where the gradient of the flow velocity distribution becomes zero. For this reason, following Earnshaw, we assume that the position where the maximum value of the flow velocity occurs and the position where the shear stress becomes zero coincide with each other;

$$
\tau_{w}=\delta \frac{d p_{w}}{d x}, \tau_{w}{ }^{\prime}=-\delta^{\prime} \frac{d p_{w}}{d x} .
$$

The four unknown variables to be obtained are $\delta, \delta^{\prime}, \tau_{w}$, and $\tau_{w}{ }^{\prime}$, and the four conditional equations are given by Eqs. (5), (6), and (7) $\left(\delta+\delta^{\prime}=G\right.$ can be used instead of Eq. (6)). Thus, we can obtain the distribution of the flow velocity between the circular pipes.

\section{1. 2 Average roughness height}

The average roughness heights, $k$ and $k^{\prime}$, are required for solving Eq. (5). In this section, by assuming that the hydraulic friction coefficient, $\lambda$, is known, we obtain the average roughness height using Nikuradse's frictional resistance formula (Nikuradse, 1950). Even though the formulas are described with respect to the average height, $k$, the formulas with respect to $k^{\prime}$ are similar. Nikuradse's frictional resistance formula for a crude pipe is given by 


$$
\frac{1}{\sqrt{\lambda}}-\frac{1}{\sqrt{8}} \frac{1}{\kappa} \ln \frac{\delta}{k}=\tilde{A}+\tilde{B} \ln \frac{u_{\tau} k}{v}
$$

where, for $\frac{u_{\tau} k}{v} \leq 4$ (hydrodynamic smooth surface),

$$
\tilde{A}=0.8, \quad \tilde{B}=\frac{1}{\sqrt{8}} \frac{1}{\kappa},
$$

for $4<\frac{u_{\tau} k}{v}<70$ (transition region),

$$
\begin{array}{ll}
\text { for } 4<\frac{u_{\tau} k}{v} \leq 7, & \tilde{A}=1.18, \tilde{B}=0.491, \\
\text { for } 7<\frac{u_{\tau} k}{v} \leq 14, & \tilde{A}=2.14, \tilde{B}=0, \\
\text { for } 14<\frac{u_{\tau} k}{v}<70, & \tilde{A}=2.81, \tilde{B}=-0.255,
\end{array}
$$

and for $\frac{u_{\tau} k}{v} \geq 70$ (rough surface),

$$
\tilde{A}=1.71, \tilde{B}=0 .
$$

Only in the first step of the repeated calculation, the average roughness height is obtained from Eq. (10) of the friction resistance formula for a rough pipe (Pope, 2000). Equation (10) is obtained by substituting Eq. (9e) into Eq. (8), as follows:

$$
\frac{\lambda}{8}=\frac{1}{\left(\frac{1}{\kappa} \ln \frac{\delta}{k}+4.84\right)^{2}}
$$

\section{1. 3 Additional constant of $\log$ law}

Additional constant $C$ (and $C^{\prime}$ ) is required for solving Eq. (5). This constant is obtained from Nikuradse's rough pipe experimental formula, which is given as follows:

For $\frac{u_{\tau} k}{v} \leq 4$ (hydrodynamic smooth surface),

$$
C=5.5+\frac{1}{\kappa} \ln \frac{u_{\tau} k}{v},
$$

for $4<\frac{u_{\tau} k}{v}<70$ (transition region),

$$
\begin{array}{ll}
\text { for } 4<\frac{u_{\tau} k}{v} \leq 7, & C=6.59+1.52 \ln \frac{u_{\tau} k}{v}, \\
\text { for } 7<\frac{u_{\tau} k}{v} \leq 14, & C=9.58, \\
\text { for } 14<\frac{u_{\tau} k}{v}<70, & C=11.5-0.704 \ln \frac{u_{\tau} k}{v},
\end{array}
$$

and for $\frac{u_{\tau} k}{v} \geq 70$ (rough surface),

$$
C=8.48 \text {. }
$$

\section{1. 4 Pressure gradient on train side surface}

The pressure gradient on the train side surface is required for solving Eq. (6). This pressure gradient, $d p_{w} / d x$, is obtained from its relationship with the flow velocity, $u$, in front of the train. In the simple calculation method, the pressure gradient is calculated using Yamamoto's one-dimensional incompressible steady-state model (Yamamoto, 1973), which is given by 


$$
\frac{d p_{w}}{d x}=-\frac{1}{2} \rho\left[\frac{\lambda}{d} \frac{(R V-u)|R V-u|}{(1-R)^{3}}+\frac{\lambda^{\prime}}{d^{\prime}} \frac{R(V-u)^{2}}{(1-R)^{3}}\right],
$$

where $u$ is the flow velocity in front of the train (the flow velocity in the region without the train), and it is obtained by

$$
\begin{aligned}
R-\frac{u}{V} & =\frac{(1-R) R\left(b_{1}-b_{2}\right)}{(1-R) b_{1}+R b_{2}+\sqrt{b_{1} b_{2}+\frac{\lambda l}{d}\left|b_{1}-b_{2}\right|}}, \\
b_{1} & =\left[\frac{\lambda}{d}(L-l)+C_{0}\right](1-R), \\
b_{2} & =\frac{1}{R}\left[(1-R)\left(C_{D p}+R\right)+\frac{\lambda^{\prime} l}{d^{\prime}}\right],
\end{aligned}
$$

where $V$ is the running speed of the train, $A$ is the tunnel cross-sectional area, $A^{\prime}$ is the train cross-sectional area, $B$ is the tunnel circumference, $B^{\prime}$ is the train circumference, $d$ is the tunnel hydraulic diameter $(=4 A / B), d^{\prime}$ is the train hydraulic diameter $\left(=4 A^{\prime} / B^{\prime}\right), \lambda$ is the tunnel hydraulic friction coefficient, $\lambda^{\prime}$ is the train hydraulic friction coefficient, $L$ is the tunnel length, $l$ is the train length, $C_{D p}$ is the train pressure drag coefficient, $C_{0}$ is the tunnel entrance/exit loss coefficient, and $R\left(=A^{\prime} / A\right)$ is the ratio between the cross-sectional areas of the train and tunnel. The sign on the right-hand side of the equation corresponding to Eq. (12) is positive in the original paper (Yamamoto, 1973). However, in this paper, because the direction of travel of the train is reversed, the sign on the righthand side of Eq. (12) is changed to negative. The model proposed in section 3.1.1 does not impose a mass conservation condition. Therefore, the spatial average flow velocity, $\bar{U}$, obtained from Eq. (15) and the train side flow velocity, $u^{\prime}-V=(u-V) /(1-R)$, observed from the train coordinate system calculated from Eq. (13a) can be different. The simple calculation method performs the following iteration calculation to correct this difference: $\alpha=\left(u^{\prime}-V\right) / \bar{U}$ is calculated, $d p_{w} / d x$ is multiplied by $\alpha^{2}$, and the loop of the simple calculation method is executed multiple times. If the number of iterations is two or more, $\alpha$ is almost one. Therefore, the number of iterations is set to two in the following calculations. The calculation program is developed using Excel VBA. The calculation time obtained for one case using Epson Endeavor (CPU: Intel Core i7) is 20-30 seconds. 


\section{Model experiment to validate the effectiveness of the simple calculation method}

\section{1 Outline of model experiment}

A model experiment was conducted to validate the effectiveness of the simple calculation method. A simple train model, equipped with a comb of total pressure tubes, was developed to measure the flow velocity around the train model. To the best of the authors' knowledge, such an experiment has been conducted for the first time internationally. Figure 5 shows an overview of the experimental equipment and a magnified view of the comb of total pressure tubes. The specifications of the train model are as follows: the length of the train model was $1.3 \mathrm{~m}$, its outer diameter was $0.06 \mathrm{~m}$, and its weight was $2.84 \mathrm{~kg}$; the nose part and the tail part were a paraboloid of revolution (aspect ratio 4), and the material was MC nylon. The length and inner diameter of the tunnel model were $12.24 \mathrm{~m}$ and $0.13 \mathrm{~m}$, respectively. The material of the tunnel was acrylic resin. The train/tunnel cross-sectional area ratio was $R=0.213$. The comb of total pressure tubes consisted of seven bulge pipes with an outer diameter of $1.1 \mathrm{~mm}$ assembling into a comb. The pressure receiving parts (pressure holes) of total pressure tubes were located at a position $0.8 \mathrm{~m}$ away from the tip of the nose of the train model. The static pressure hole for determining the flow velocity was set at a position $0.8 \mathrm{~m}$ away from the tip of the nose of the train. The static pressure hole was located on the surface of the train model on the opposite side of the comb of total pressure tubes. The flow velocity $U$ around the train model was calculated by the following equation:

$$
U=\sqrt{\frac{2\left(p_{\text {total }}-p_{\text {static }}\right)}{\rho}},
$$

where $p_{\text {total }}$ is the total pressure measured by the comb of total pressure tubes, $p_{\text {static }}$ is the static pressure measured at the static pressure hole, and $\rho$ is the air density. The minimum distance between the pressure hole in the comb of total pressure tubes and the train model surface was $2 \mathrm{~mm}$, and the maximum distance was $17 \mathrm{~mm}$. The total pressure tubes were installed in two rows, and the interval between the bulge pipes in each row was $5 \mathrm{~mm}$ in the radial direction of the train model. The positions of the respective bulge pipes from the train model surface were $2.0 \mathrm{~mm}, 4.5 \mathrm{~mm}, 7.0$ $\mathrm{mm}, 9.5 \mathrm{~mm}, 12.0 \mathrm{~mm}, 14.5 \mathrm{~mm}$, and $17 \mathrm{~mm}$, respectively. The bulge pipe and the pressure sensor were connected by a plastic tube with an inner diameter of $1.4 \mathrm{~mm}$. The distance from the pressure hole to the sensor (including both the bulge pipe and plastic tube length) was $0.105 \mathrm{~m}$. The pressure sensor used was All Sensors 20 INCH-D2-4V-MINI P2NS (operating range: $\pm 4980 \mathrm{~Pa}$, output range: $\pm 2 \mathrm{~V}$, zero point: $2.25 \mathrm{~V}$, response frequency: $2 \mathrm{kHz}$ ) (see Fig. 6). The data logger used was the Tess ultra-small data logger (sampling frequency: $5 \mathrm{kHz}$, recording time: $16 \mathrm{~s}$, number of data points: 80 thousand) (see Fig. 6).

The equipment used for the model experiment is illustrated schematically in Fig. 7. The model launcher consisted of four pairs of rotating wheels and projected an axisymmetric model train into a circular cylindrical tunnel made of transparent acrylic. The model train was guided along a stretched piano wire. Because the tunnel central axis coincided with the piano wire, the train model ran along the center of the tunnel. The model launcher used was designed for an axisymmetric train model. The model launcher accelerates the train model by sandwiching it with upper and lower rotating wheels. Therefore, the train model carrying the comb of total pressure tubes cannot be launched by the original mechanism of the model launcher. Saito et al. developed a mechanism to launch such models (Saito et al., 2014). In the newly developed mechanism, the axisymmetric thruster accelerated by rotating wheels and a pushing bar whose outside diameter was smaller than that of the thruster were connected. The train model was pushed by the pushing bar (see Fig. 7). When the thruster was accelerated by the rotating wheels, the pushing bar and the train model were also accelerated. Subsequently, the thruster and the pushing bar were stopped by the thruster braking device. Furthermore, the train model left the pushing bar, traveled with inertia, and stopped at the train model braking device.

The tunnel entrance was set up at a position approximately $10.6 \mathrm{~m}$ away from the model launcher stand. A coil (front side) for speed measurement was installed at a position $0.5 \mathrm{~m}$ away from the tunnel entrance. The distance between the front and rear coils was $2 \mathrm{~m}$. Small neodymium permanent magnets were inserted into the nose part of the model train. The train model speed was measured by detecting the magnetic field generated when the train nose passed through each of the two wire loops (see Fig. 7) placed $2 \mathrm{~m}$ apart at the rear of the tunnel entrance. The error of the speed measurement was estimated to be less than $1 \%$ (Howe et al., 2000).

The projecting speed of the model train increased at an interval of $20 \mathrm{~km} / \mathrm{h}$ from $110 \mathrm{~km} / \mathrm{h}$ to $150 \mathrm{~km} / \mathrm{h}$, and the measurement was performed three times at each speed. In the following section, only the results at $150 \mathrm{~km} / \mathrm{h}$ are shown owing to limited space. 


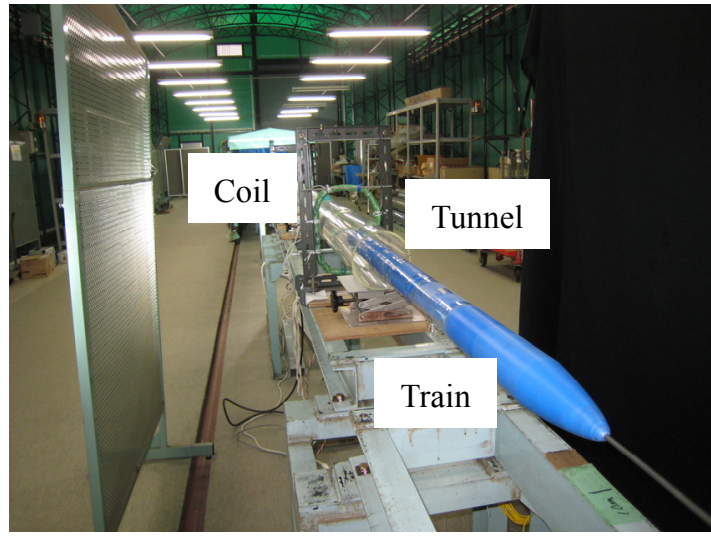

Overview of experimental equipment

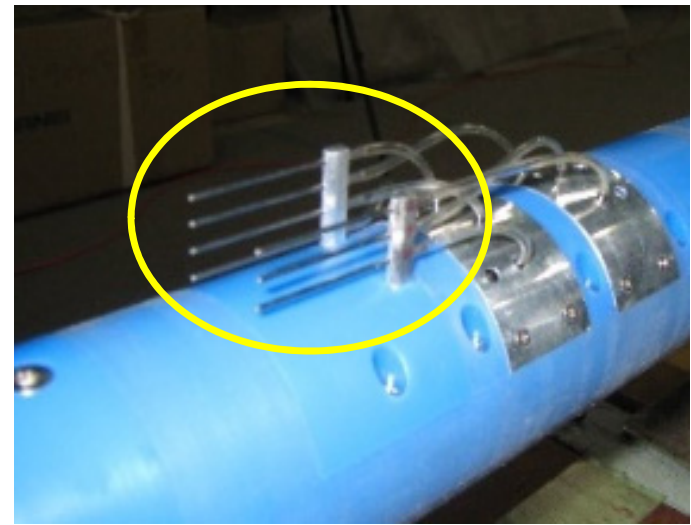

Magnified view of comb of total pressure

Fig. 5 Experimental equipment and comb of total pressure tubes

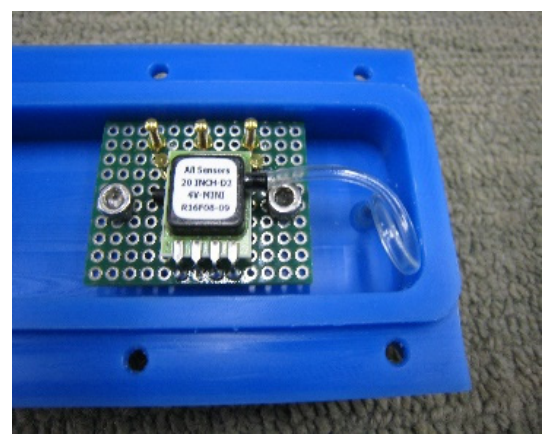

Pressure sensor (All Sensors 20INCH-D2-4V-MINI P2NS)

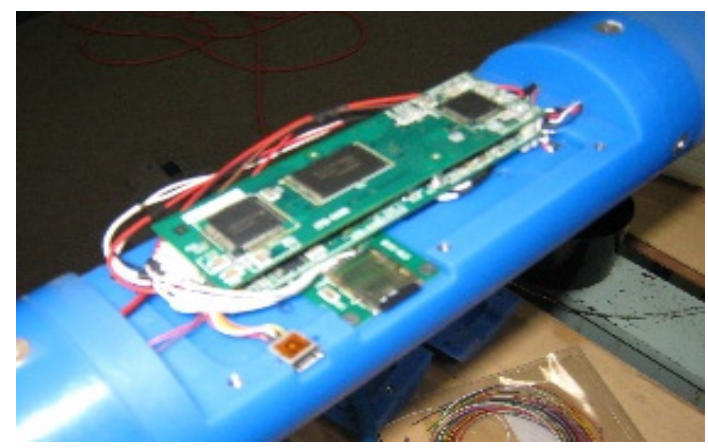

Data logger

(Tess ultra-small data logger)

Fig. 6 Pressure sensor and data logger

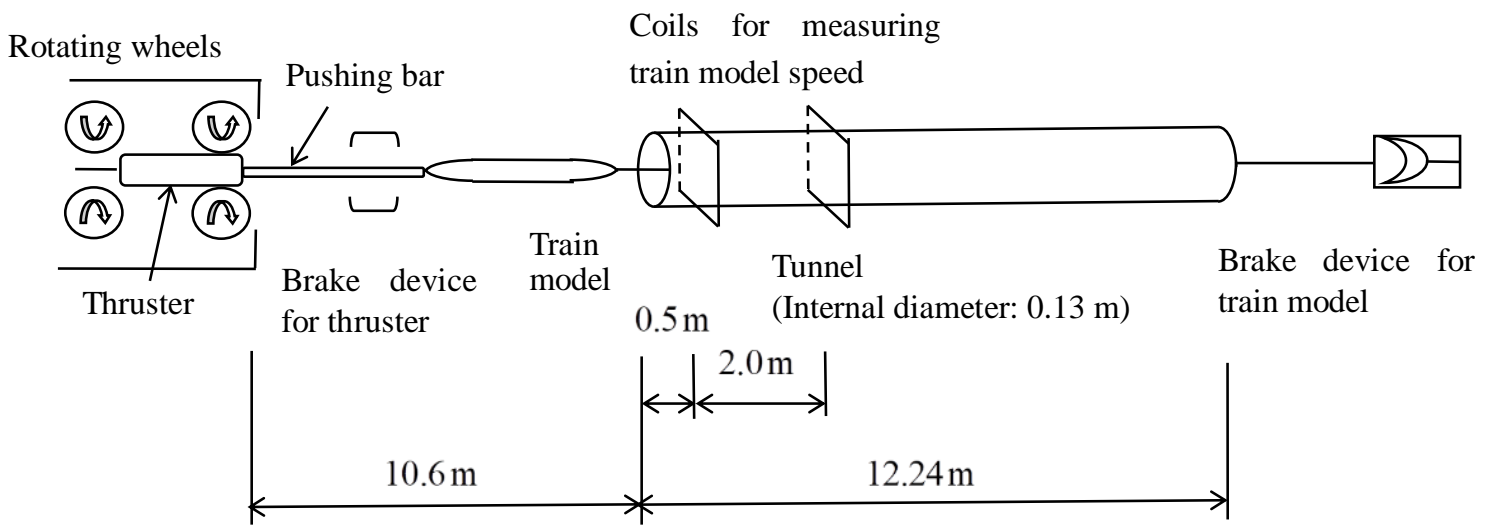

Fig. 7 Schematic of train model experimental equipment 


\section{2 Comparison of the model experiment and simple calculation method results}

The calculations for the model experiment were carried out using the simple calculation method. Table 1 shows the specifications used for the calculations. The values for the cross-sectional areas, circumferences, and lengths correspond to those of the train model and the tunnel model used in the model experiments. The hydraulic friction coefficient of the train model was estimated from the result of the model experiments that were separately carried out using Clauser's method (Kikuchi, K., et al., 2017). The materials of the train model and tunnel model were MC nylon and acrylic resin, respectively. We judged that both the surfaces could be regarded as equivalent hydrodynamic smooth surfaces, and we applied the value of the train hydraulic friction coefficient, $\lambda^{\prime}$, as that of the tunnel hydraulic friction coefficient, $\lambda$. The results are shown in Fig. 8. Figure 8 shows the flow velocity distribution in the vertical direction with the train roof as the origin. Figure 8 also shows the results of the model experiments. The results of the model experiments correspond to those of the train model just after entering the tunnel and just before exiting from the tunnel. As can be seen from Fig. 8 , the flow velocity distribution obtained by the simple calculation method reproduced the experimental results well. However, the results of the simple calculation method differ from the experimental results in two respects. First, the boundary layer thickness is only approximately $12 \mathrm{~mm}$ in the experimental results, although the simple calculation method assumes a fully developed turbulent boundary layer in the entire flow field. The boundary layer was not sufficiently developed at the measurement point in the model experiment. Second, the flow velocity obtained using the simple calculation method is smaller than that obtained using the model experiment. The reason why the flow velocity distribution obtained by the simple calculation method becomes smaller than that obtained by the model experiment is considered to be as follows: The simple calculation method assumes a situation where sufficient time has passed after the train enters the tunnel. This situation was not reproduced in the model experiment, even at the time just before the train model exited from the tunnel. Despite these discrepancies, the results obtained by the simple calculation method reproduced the experimental results well. These discrepancies can be resolved by conducting experiments using a longer train model in the model experiment and by extending the train side pressure gradient to an unsteady-state model in the simple calculation method. However, these matters will be the subjects of future investigations.

As mentioned above, the analytical model was built on the assumption that the boundary layer in the annulus develops sufficiently. However, as the length of the train model used in the experiment was short, the boundary layer did not develop sufficiently at the position where flow velocity was measured. Therefore, there is a difference between the calculation results and experimental results. We used two indices to evaluate the magnitude of the difference quantitatively. One is the maximum flow velocity, $\langle U\rangle_{\max }$, between the two circular pipes, and the other is the spatial average flow velocity, $\bar{U}$, between the two circular pipes, which is defined by

$$
\bar{U}=\frac{1}{G} \int_{0}^{G}\langle U\rangle d y \text {. }
$$

Table 2 lists the normalized maximum flow velocity and the normalized spatial average flow velocity obtained by the simple calculation method. For comparison, the flow velocities obtained by the model experiment are also listed. The experimental results show the values just after the train entered the tunnel and those just before the train model exited the tunnel. From Table 2, it can be seen that the normalized maximum flow velocity and the normalized spatial average flow velocity obtained by the simple calculation method are smaller than those obtained by the model experiment. However, the ratio of the flow velocities obtained by the simple calculation method to that obtained by the model experiment is $\sim 0.8-0.9$, and the ratio meets the original target calculation accuracy of $70-80 \%$. It is expected that calculation accuracy will be improved if the data measured under the condition that the boundary layer develops sufficiently are obtained. If we analyze the flow around the train running in the tunnel by the three-dimensional numerical fluid simulation, the time required for the analysis is approximately 20 hours (Cray XC30, 13 cores parallel computation). However, the calculation time of the simple calculation method is 20-30 seconds (Epson Endeavor, CPU: Intel Core i7). Accordingly, the simple calculation method is suitable for examining the influence of changing parameters of the train and the tunnel. Next, we examine the influence of changing the hydraulic frictional coefficients on the train roof flow in the following section. 
Kikuchi, Noguchi, Nakade and Mashimo, Mechanical Engineering Journal, Vol.5, No.3 (2018)

Table 1 Specifications of calculation (basic condition values)

\begin{tabular}{|c|c|c|c|c|}
\hline Classification & Items & Symbols & Units & Values \\
\hline \multirow[t]{5}{*}{ Tunnel } & Cross-sectional area & $A$ & $\mathrm{~m}^{2}$ & 0.01327 \\
\hline & Circumference & $B$ & $\mathrm{~m}$ & 0.4084 \\
\hline & Length & $L$ & $\mathrm{~m}$ & 12.24 \\
\hline & Hydraulic friction coefficient & $\lambda$ & - & 0.0156 \\
\hline & Entrance/exit loss coefficient & $C_{0}$ & - & 1.0 \\
\hline \multirow[t]{6}{*}{ Train } & Cross-sectional area & $A^{\prime}$ & $\mathrm{m}^{2}$ & 0.002827 \\
\hline & Circumference & $B^{\prime}$ & $\mathrm{m}$ & 0.1885 \\
\hline & Length & $l$ & $\mathrm{~m}$ & 1.3 \\
\hline & Hydraulic friction coefficient & $\lambda^{\prime}$ & - & 0.0156 \\
\hline & Pressure drag coefficient & $C_{D p}$ & - & 0.10 \\
\hline & Running speed & $V$ & $\mathrm{~km} / \mathrm{h}$ & 150 \\
\hline \multirow{3}{*}{$\begin{array}{l}\text { Various } \\
\text { coefficients }\end{array}$} & Air density & $\rho$ & $\mathrm{kg} / \mathrm{m}^{3}$ & 1.225 \\
\hline & Kinematic viscosity coefficient & $v$ & $\mathrm{~m}^{2} / \mathrm{s}$ & $1.51 \times 10^{-5}$ \\
\hline & Karman constant & $\kappa$ & - & 0.41 \\
\hline
\end{tabular}

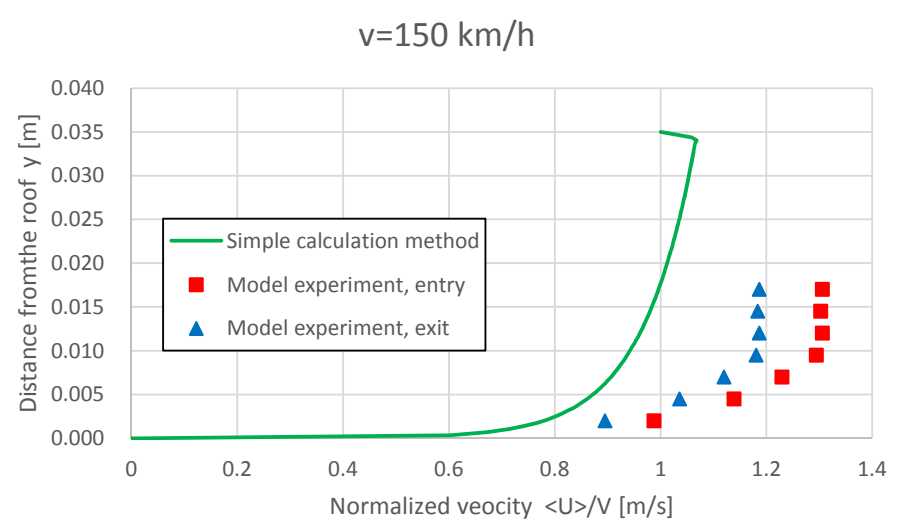

Fig. 8 Comparison of simple calculation method and model experimental results

Table 2 Normalized maximum flow velocity and normalized spatial average flow velocity

\begin{tabular}{|c|c|c|c|c|c|}
\hline & \multirow{2}{*}{$\begin{array}{c}\text { Simple } \\
\text { calculation } \\
\text { method }\end{array}$} & \multicolumn{2}{|c|}{ Model experiment } & \multicolumn{2}{|c|}{$\begin{array}{c}\text { Simple calculation method } \\
\text { / Model experiment }\end{array}$} \\
\cline { 3 - 6 } & & Entry & Exit & Entry & \multicolumn{2}{|c|}{ Exit } \\
\hline $\begin{array}{c}\text { Normalized maximum flow velocity } \\
\langle U\rangle_{\max } / V\end{array}$ & 1.07 & 1.31 & 1.19 & 0.82 & 0.90 \\
\hline $\begin{array}{c}\text { Normalized spatial average flow } \\
\text { velocity } \\
\bar{U} / V\end{array}$ & 0.96 & 1.23 & 1.12 & 0.78 & 0.86 \\
\hline
\end{tabular}




\section{Influence of some parameters on train roof flow velocity}

In the simple calculation method, the values of various parameters are required to perform the calculation. Here, we examine the influence of hydraulic friction coefficients on the train roof flow velocity.

\subsection{Influence of tunnel hydraulic friction coefficient}

Calculations were performed by changing the tunnel hydraulic friction coefficient in order to examine its influence on the train roof flow velocity. The flow velocity distributions are shown in Fig. 9. To enhance the clarity of the figure, the range of the horizontal axis in Fig. 9 is enlarged from that used in Fig. 8. Table 3 lists the results of the normalized reference point flow velocity, normalized spatial average flow velocity, tunnel wall shear stress, and train roof shear stress. The reference point was located at $y_{0}=G / 2=0.0175 \mathrm{~m}$.

From the flow velocity distribution in Fig. 9, it can be seen that the maximum flow velocity at the contact point between the train roof boundary layer and the tunnel wall boundary layer increases with the tunnel hydraulic friction coefficient. From the results in Table 3, it can be seen that both the normalized reference point flow velocity and the normalized spatial average flow velocity increase with the tunnel hydraulic friction coefficient. The normalized reference point flow velocity is larger than the normalized spatial average flow velocity (corresponding to the flow velocity of the one-dimensional pressure variation simulation). Therefore, even with the simple calculation method, it is possible to investigate the tendency of the velocity at the reference point to be larger than the spatial average flow velocity due to the effect of the boundary layer.

The ratio of the tunnel wall velocity gradient to that of the train roof is defined as the wall velocity gradient ratio, $\varphi$ (El Telbany, 1980):

$$
\varphi=\frac{\mu(\partial\langle U\rangle / \partial y)_{y=G}}{\mu(\partial\langle U\rangle / \partial y)_{y=0}}=\frac{\tau_{w}}{\tau_{w}{ }^{\prime}} .
$$

In the simple calculation method, the log law is assumed as the flow velocity distribution. The train roof flow velocity distribution varies with the value of the wall velocity gradient ratio $\varphi$. The schematic diagrams of the relationship between the values of the wall velocity gradient ratio and the train roof flow velocity distribution are shown in Fig. 10. When $\varphi=-1$, the train roof flow velocity distribution is a Poiseuille flow driven by a pressure gradient. In contrast, when $\varphi=1$, the train roof flow velocity distribution is a Couette flow driven by a frictional force with the pressure gradient being zero. When $-1<\varphi<1$, the train roof flow velocity distribution is a combination of Poiseuille flow and Couette flow. In this study, such a mixed flow is further classified into three types: a Poiseuille mixed flow when $-1<\varphi<0$, a neutral mixed flow when $\varphi=0$, and a Couette mixed flow when $0<\varphi<1$. Furthermore, if the pressure gradient is weakly adverse, $\varphi>1$, which is referred to as an odd Couette mixed flow in this paper. As is obvious from Eq. (16), when $\varphi<0, \tau_{w}$ and $\tau_{w}{ }^{\prime}$ have different signs, and when $\varphi>0, \tau_{w}$ and $\tau_{w}{ }^{\prime}$ have the same sign.

From Table 3, it can be seen that the absolute values of both the wall velocity gradient of the train roof boundary layer and that of the tunnel wall boundary layer increase with the tunnel hydraulic friction coefficient (wall shear stresses are shown instead of wall velocity gradients in Table 3). Figure 11 shows the variation in wall velocity gradient ratio when the tunnel hydraulic friction coefficient is changed. From Fig. 11, it can be seen that the wall velocity gradient ratio is in the range of $-1<\varphi<0$, and the train roof flow velocity distribution considered in the simple calculation method is a Poiseuille mixed flow. This is obvious from the assumption of the simple calculation method. As can been seen from Eq. (7), $\tau_{w}$ and $\tau_{w}{ }^{\prime}$ have different signs and $\varphi<0$. In order to investigate the flow velocity distribution when $\varphi>0$, Eq. (7) needs to be modified as follows.

$$
\frac{\partial\langle U\rangle}{\partial y}=\frac{u_{\tau}}{\kappa} \frac{k}{\delta}=\frac{u_{\tau}^{\prime}}{\kappa} \frac{k^{\prime}}{\delta^{\prime}}
$$

However, this situation is not handled by the simple calculation method yet. From Fig. 11, it is understood that the absolute value of the wall velocity gradient ratio decreases with tunnel hydraulic friction coefficient. Furthermore, as the tunnel hydraulic friction coefficient approaches zero, the wall shear stress ratio also approaches zero. As a result, the train roof flow becomes a neutral mixed flow. 


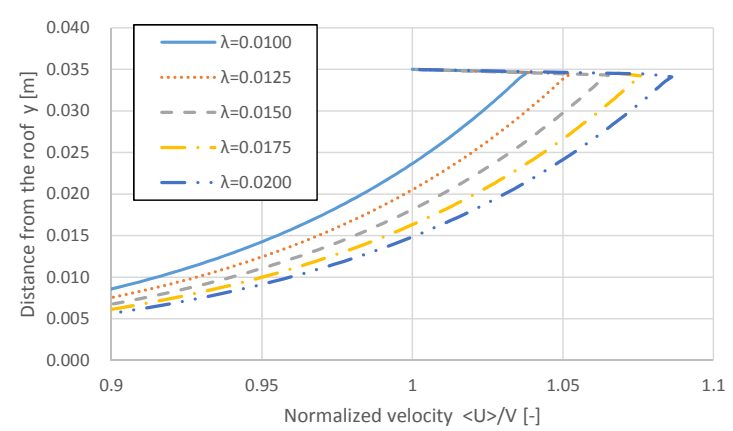

Fig. 9 Variation in flow velocity distribution with tunnel hydraulic friction coefficient

Table 3 Influence of tunnel hydraulic friction coefficient on each flow velocity

\begin{tabular}{|c|c|c|c|c|}
\hline $\begin{array}{c}\text { Tunnel hydraulic } \\
\text { friction coefficient }\end{array}$ & $\begin{array}{c}\text { Normalized reference } \\
\text { point flow velocity } \\
\lambda U\rangle_{y=y_{0}} / V\end{array}$ & $\begin{array}{c}\text { Normalized spatial } \\
\text { average flow velocity } \\
\bar{U}\end{array}$ & $\begin{array}{c}\text { Tunnel wall shear } \\
\text { stress [Pa] } \\
\tau_{w}=\mu(\partial\langle U\rangle / \partial y)_{y=G}\end{array}$ & $\begin{array}{c}\text { Train roof shear stress } \\
{[\mathrm{Pa}]}\end{array}$ \\
\hline$\tau_{w}{ }^{\prime}=\mu(\partial\langle U\rangle / \partial y)_{y=0}$ \\
\hline 0.0100 & 0.970 & 0.927 & -0.038 & 3.48 \\
\hline 0.0125 & 0.984 & 0.941 & -0.055 & 3.57 \\
\hline 0.0150 & 0.996 & 0.952 & -0.071 & 3.65 \\
\hline 0.0175 & 1.007 & 0.962 & -0.087 & 3.73 \\
\hline 0.0200 & 1.017 & 0.972 & -0.102 & 3.79 \\
\hline
\end{tabular}

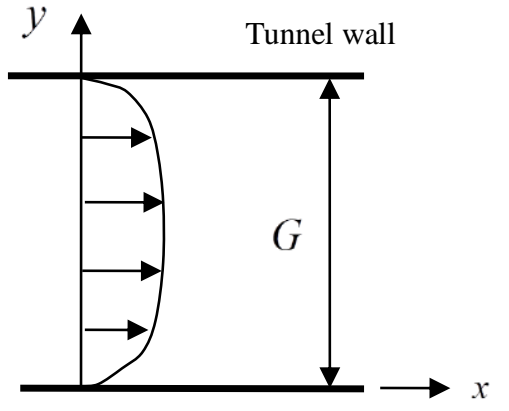

Train roof

$\varphi=-1$

(a) Poiseuille flow (pressure gradient drive)

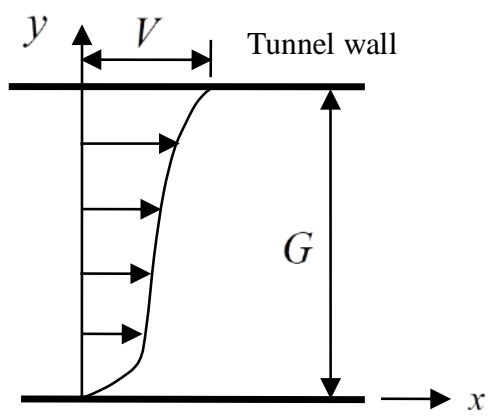

Train roof

$$
0<\varphi<1
$$

(d) Couette mixed flow

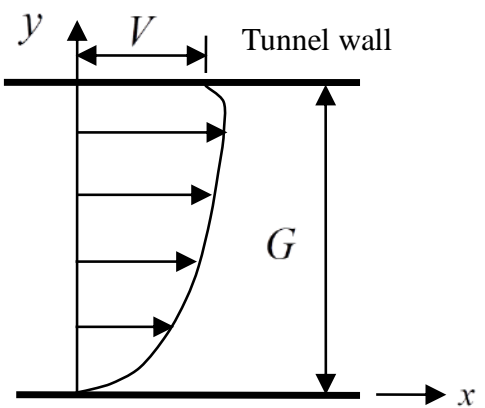

Train roof $-1<\varphi<0$

(b) Poiseuille mixed flow

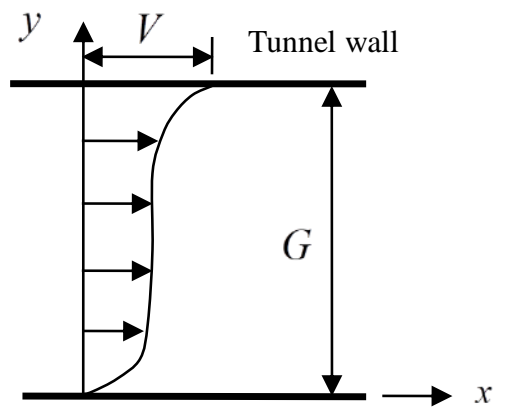

Train roof

$$
\varphi=1
$$

(e) Couette flow (friction force drive)

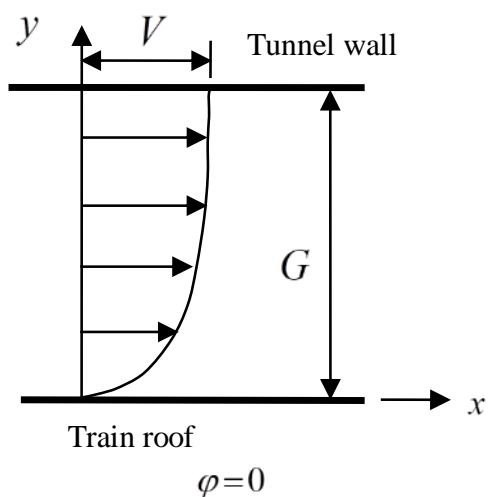

(c) Neutral mixed flow

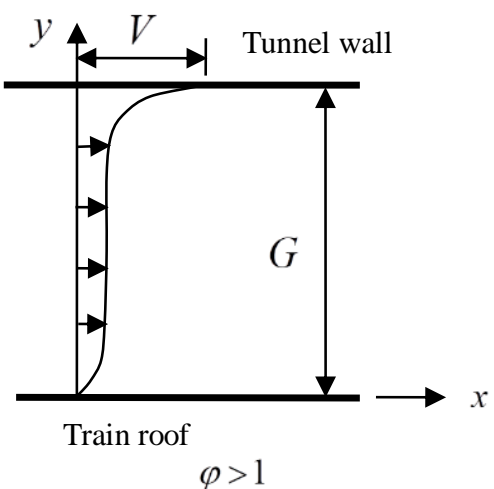

(f) Odd Couette mixed flow

Fig. 10 Schematic diagrams of the relationship between the wall velocity gradient ratio and train roof flow velocity distribution 


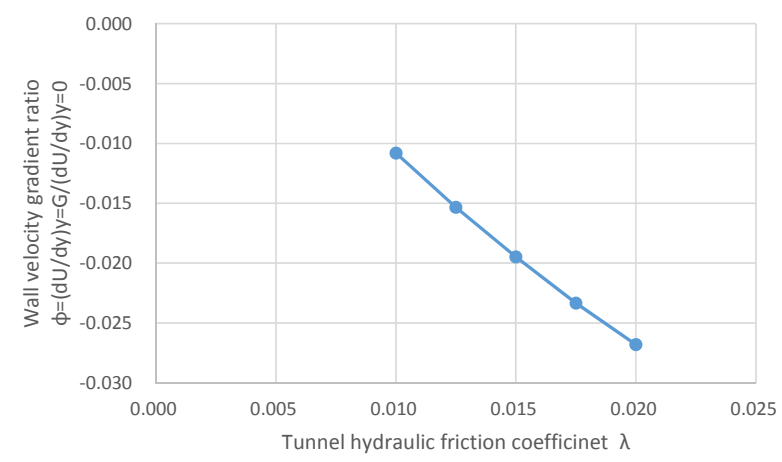

Fig. 11 Variation in flow velocity gradient ratio with tunnel hydraulic friction coefficient

\section{2 Influence of train hydraulic friction coefficient}

Next, calculations were performed by changing the train hydraulic friction coefficient to examine its effect on the train roof flow velocity. The flow velocity distributions are shown in Fig. 12, and Table 4 lists the results of the normalized reference point flow velocity, normalized spatial average flow velocity, tunnel wall shear stress, and train roof shear stress.

From the flow velocity distribution shown in Fig. 12, it can be seen that the maximum flow velocity at the contact point between the train roof boundary layer and the tunnel wall boundary layer decreases with the train hydraulic friction coefficient. From the results in Table 4, it can be seen that with increase in train hydraulic friction coefficient, both the normalized reference point flow velocity and the normalized spatial average flow velocity decrease, in contrast to the case in which the tunnel hydraulic friction coefficient increases. Moreover, the normalized reference point flow velocity is larger than the normalized spatial average flow velocity (corresponding to the flow velocity of the one-dimensional pressure variation simulation).

From Table 4, it can be seen that the absolute values of both the velocity gradient of the train roof boundary layer and that of the tunnel wall boundary layer decrease as the train hydraulic friction coefficient increases (wall shear stresses are shown instead of velocity gradients in Table 4). Figure 13 shows the variation in wall velocity gradient ratio when the train hydraulic friction coefficient is changed. From Fig. 13, it is understood that the wall velocity gradient ratio is in the range of $-1<\varphi<0$, and the train roof flow velocity distribution in this case is a Poiseuille mixed flow. Furthermore, it can be seen that as the train hydraulic friction coefficient increases, the absolute value of the wall flow gradient ratio decreases and approaches zero, in contrast to the case in which the tunnel hydraulic friction coefficient is changed. 


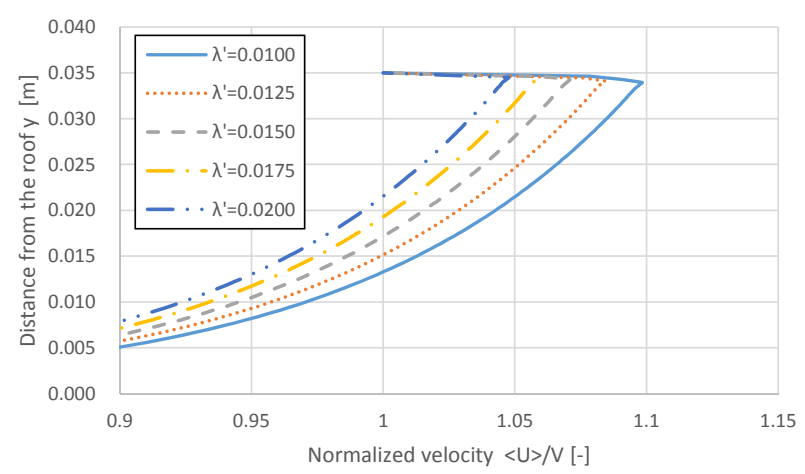

Fig. 12 Variation in flow velocity distribution with train hydraulic friction coefficient

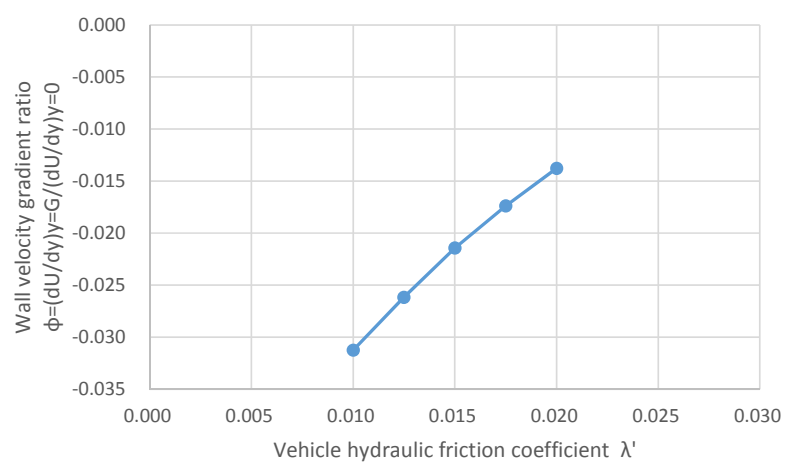

Fig. 13 Variation in flow velocity gradient ratio with train hydraulic friction coefficient

Table 4 Influence of train hydraulic friction coefficient on each flow velocity

\begin{tabular}{|c|c|c|c|c|}
\hline $\begin{array}{c}\text { Train hydraulic } \\
\text { friction coefficient } \\
\lambda^{\prime}\end{array}$ & $\begin{array}{c}\text { Normalized reference } \\
\text { point flow velocity } \\
\langle U\rangle_{y=y_{0}} / V\end{array}$ & $\begin{array}{c}\text { Normalized spatial } \\
\text { average flow velocity } \\
\bar{U} / V\end{array}$ & $\begin{array}{c}\text { Tunnel wall shear } \\
\text { stress [Pa] } \\
\tau_{w}=\mu(\partial\langle U\rangle / \partial y)_{y=G}\end{array}$ & $\begin{array}{c}\text { Train roof shear stress } \\
{[\mathrm{Pa}]}\end{array}$ \\
\hline 0.0100 & 1.029 & 0.983 & -0.121 & $3(\partial\langle U\rangle / \partial y)_{y=0}{ }^{\prime}=$ \\
\hline 0.0125 & 1.015 & 0.970 & -0.099 & 3.87 \\
\hline 0.0150 & 1.002 & 0.958 & -0.079 & 3.69 \\
\hline 0.0175 & 0.990 & 0.946 & -0.063 & 3.61 \\
\hline 0.0200 & 0.979 & 0.936 & -0.049 & 3.54 \\
\hline
\end{tabular}

\section{Conclusions}

The flow velocity on a roof of a train running in a tunnel is an important factor in examining the current collection characteristics of a pantograph. Therefore, in this study, we proposed a simple calculation method that enabled us to easily calculate the flow on the roof of a train running in a tunnel using a personal computer. Using the proposed simple calculation method and a personal computer, we could quickly and easily calculate flow rate with a calculation accuracy equivalent to that of the one-dimensional pressure fluctuation simulation. In order to validate the effectiveness of the proposed simple calculation method, a model experiment was carried out, and the calculation results and experimental results were compared. Furthermore, the effects of hydraulic friction coefficients on the train roof flow velocity were examined by using the simple calculation method, which was not time consuming. The results of this study are summarized as follows.

(1) To calculate the flow velocity on the roof of a train running in a tunnel using a personal computer, we employed Earnshaw's method and proposed a simple calculation method. Nikuradse's frictional resistance formula was used for the evaluation of the average roughness height, and Yamamoto's one-dimensional incompressible steady-state model was used to evaluate the train side surface pressure gradient. The target calculation accuracy was set as approximately $70-80 \%$ of the experimental results. With the proposed simple calculation method, the calculation time for one case was as short as 20-30 seconds.

(2) A model experiment was conducted to validate the effectiveness of the proposed simple calculation method, and the results of the model experiment and the simple calculation method were compared. Although the normalized velocities obtained by the simple calculation method were smaller than those of the model experiment, the calculation accuracy was approximately $80-90 \%$, which met the original target calculation accuracy.

(3) The effects of the tunnel hydraulic friction coefficient and train hydraulic friction coefficient on the train roof flow 
were examined using the proposed simple calculation method. The tunnel hydraulic friction coefficient and train hydraulic friction coefficient were found to have opposite effects on the normalized reference point flow velocity, normalized special average flow velocity, and wall velocity gradient ratio.

In the model experiment conducted in this study, the flow velocity measurement point was located at a position not far from the tip of the nose of the train model; hence, the flow velocity distribution did not become a fully developed turbulent boundary layer. In future, we are planning to conduct experiments using longer train models. In the simple calculation method, Yamamoto's steady-state model was used to calculate the train side pressure gradient. To further improve the estimation accuracy, it is necessary to extend the steady-state model to the unsteady-state model.

At the end, we would like to express our sincere gratitude to the reviewers and the associate editor for their useful and beneficial suggestions and comments presented through the careful review of this paper.

\section{References}

El Telbany, M. M. M., and Reynolds, A. J., Velocity Distributions in Plane Turbulent Channel Flows, Journal of Fluid Mechanics, Vol. 100 (1980), pp. 1-29

Earnshaw, P. B., On the Aerodynamic Resistance to Long Trains Passing Through Long Close-fitting Tunnels, Royal Aircraft Establishment, Technical Report 70199 (1970), pp. 1-56

Flueckiger, M., Reinke, P., Nyfeler, S., and Kapfenberger, W., Contact Force Disturbances of the Pantograph System due to Air Flow at High-speed Trains in Tunnels, ISAVFT 15, BHR Group (2013), pp. 763-776

Howe, M., Iida, M., Fukuda, T., and Maeda, T., Theoretical and Experimental Investigation of the Compression Wave Generated by a Train Entering a Tunnel with a Flared Portal, Journal of Fluid Mechanics, Vol. 425 (2000), pp. 111132

Iida, M., and Maeda, T., Numerical Calculation of Pressure Transients in Railway Tunnel, RTRI Report, Vol. 4, No. 7 (1990), pp. 54-62 (in Japanese)

Ikeda, M., Mitsumoji, T., and Yamashita, Y., Direction and Velocity Characteristics of Air Flow around Pantograph of Running Train, RTRI Report, Vol. 25, No. 6 (2011), pp. 11-16 (in Japanese)

Ikeda, M., Research Trends on Current Collection Performance, Rolling Stock \& Technology, No. 200 (2013), pp. 2-8 (in Japanese)

Kajiyama, H., Iida, M., Maeda, T., Murata, K., Ido, A., and Fukuda, T., Numerical Calculation of Pressure Transients in Railway Tunnel System, Aerodynamics and Ventilation of VEH, BHR Group (1994), pp. 1-13

Kajiyama, H., Numerical simulation of thermal environment in underground railways, Aerodynamics and Ventilation of Vehicle and Tunnel, BHR Group (2003), pp. 809-816

Kikuchi, K., and Noguchi, Y., Measurement of flow velocity by bomb of total pressure tubes mounted on simple shape train model running in the open section, Annual meeting of the Japan Society of Fluid Mechanics 2017, USB (2017)

Manabe, K., High-Speed Contact Performance of Catenary-Pantograph System, Transactions of the Japan Society of Mechanical Engineers, Series C, Vol. 54, No. 504 (1988), pp. 1843-1851 (in Japanese)

Minegishi, A., Tanaka, K., Takei, Y., and Izumi, Y., Development of "Pressure Variation Forecast System of Station According to High Speed Train" and Application, J-Rail2008 (2008), pp. 635-638 (in Japanese)

Mitsumoji, T., Ikeda, M., and Sato, Y., Clarification of Lift Force Variation Mechanism of a Pantograph Head of Shinkansen, RTRI Report, Vol. 30, No. 2 (2016), pp. 11-16 (in Japanese)

Nikuradse, Law of Flow in Rough Pipes, NACA TM 1292 (Translation of "Ströemungsgesetze in rauhen Rohren." 1933) (1950), pp. 1-62

Noguchi, Y., Kikuchi, K., Nakade, K., and Mashimo, S., Numerical Analysis of Flow over the Roof of the Train Running in the Tunnel, Mechanical Engineering Congress, Japan, G1800204 (2015), CD-ROM (in Japanese)

Pope, S. B., Turbulent Flows (2000), pp. 264-332, Cambridge University Press

Saito, S., Iida, M., and Kajiyama, H., Numerical Simulation of 1-D Unsteady Compressible Flow in Railway Tunnels, Journal of Environment and Engineering, Vol. 6, No. 4 (2011), pp. 723-738

Saito, S., and Fukuda, T., An experimental investigation on pressure variation during train passage in tunnel, Transactions of the JSME, Vol. 80, No. 819 (2014), pp. 1-14 (in Japanese) 
Suzuki, M., Ikeda, M., and Koyama, T., Flow Control for Pantographs Using Air Intake and Outlet, Journal of Mechanical Systems for Transportation and Logistics, Vol. 1, No. 3 (2008), pp. 272-280

Yamamoto, A., Aerodynamics of Train and Tunnel, Proceedings of the First International Conference on Vehicle Mechanics (1968), pp. 151-163

Yamamoto, N., Pressure Variations, Aerodynamic Drag and Tunnel Ventilation in Shin-Kan-Sen Type Tunnel, Railway Technical Research Report, No. 871 (1973), pp. 60-74 (in Japanese)

Yamauchi, Y., Saito, S., and Kajiyama, H., Numerical Calculation Results of Pressure Variation in a Railway Tunnel, RTRI Report, Vol. 30, No. 7 (2016), pp. 47-52 (in Japanese) 